\title{
INTERNET SKILLS, SOURCES OF SUPPORT AND BENEFITING FROM INTERNET USE
}

\author{
Alexander J.A.M. van Deursen ${ }^{1}$, Cedric Courtois ${ }^{2}$ and Jan A.G.M. van Dijk ${ }^{1}$
}

1. University of Twente, the Netherlands.

2. iMinds-MICT-Ghent University, Belgium.

Alexander J.A.M. van Deursen (Corresponding Author)

University of Twente / Faculty of Behavioural Sciences

Department of Media, Communication and Organization

Cubicus Building

PO Box 217

7500 AE Enschede

The Netherlands

a.j.a.m.vandeursen@utwente.nl

Phone: +31534891021 (-3299 secr.)

Fax: +31534894259

\begin{abstract}
This study added communication Internet skills to an existing skill framework of operational, formal, information and strategic skills. We investigated how people deal with inadequate skill levels by identifying support sources. Furthermore, we investigated which of the Internet skills actually matter for attaining beneficial Internet outcomes and whether support sources employed moderate these effects. Results of a large scale survey revealed three support patterns: independents, social support seekers and formal help seekers. The newly added communication skills prove to be an important addition since they have an independent effect on beneficial Internet use. The group of independent Internet users benefited more from Internet use than formal help seekers and much more than social support seekers. Internet communication skills hold the potential for achieving a high degree of independence in using the Internet by compensating for information skills so as to attain beneficial Internet outcomes.
\end{abstract}




\section{INTRODUCTION}

Digital skills have been marked as an important factor in explaining differences in individuals' Internet use (e.g., Mossberger, Tolbert \& Stansbury, 2003; Norris, 2001; Solomon, Allen \& Resta, 2003; Warschauwer, 2003; Van Dijk, 2005). These skills are relatively novel with regard to the digital divide debate and there has been little work on the online abilities of the average Internet user (Hargittai \& Hinnant, 2008). Existing empirical investigations point towards large differences in skill levels between segments of the population. Recently, Van Deursen \& Van Dijk (2009, 2010) proposed a range of Internet skills that would combine several digital skill conceptualizations. Their definition accounts for technical or media aspects (medium-related skills) and substantial or content aspects (content-related skills). Medium-related Internet skills consist of operational skills, which include a basic command of an Internet browser, and formal skills, which include the ability to navigate and orient oneself within the Internet's hypermedia structure. The first type of content-related Internet skills consists of information skills, which include the ability to find, select and evaluate sources of information on the Internet. Secondly, strategic skills refer to one's capacity to use the Internet as a means to reach particular personal and professional goals. This and other conceptualizations of Internet skills have thus far mainly focused on the information function of the Internet. The first contribution of this study is the addition of communication Internet skills. A few scholars have suggested to add these skills to existing literacy frameworks (e.g., Ba, Tally \& Tsikalas, 2002; Eshet Alkalai \& Amichai Hamburger, 2004; Lankshear \& Knobel, 2008; Livinstone, 2008), however the exact definition varies. Internet use requires a distinct set of skills that allow one to effectively cope with this medium that generally is deprived of rich verbal cues (e.g., speech intonation) and visual cues (e.g., smiles and nodding). It is necessary to become accustomed to patterns of asynchronous communication and to the flood of messages that is largely unavailable in traditional media. More specifically, people have to learn how to cope with the complexity of instant messaging, social networking and other available online communication applications.

When someone has an inadequate level one of the aforementioned Internet skills, there are distinctive ways to deal with this. One way is to attempt to resolve this lack of proficiency through trial and error, whereas another is to rely on various kinds of support sources. For example, someone who lacks the basic skill of operating a browser might ask a relative for assistance or may eventually rely on more formal support, such as an Internet course, to obtain solid training. Besides adding communication skills to the Internet skills definition, this study examines the relationship between Internet skills and sources of support. More specifically, we investigate distinct patterns of soliciting support sources $(R Q 1)$ and examine how these patterns relate to mean levels of operational, formal, information, communication and strategic skills (RQ2). Presently, there are many means to an end when using the Internet, which implies that one skill can be used to compensate for another skill.

After defining and extending the skills framework and focusing on support sources, we continue with discussing how this all actually matters. When people lack proficiency in one of the aforementioned Internet skills, this might exclude them from beneficial outcomes 
the Internet has to offer. Therefore, we question which Internet skills actually matter for attaining beneficial Internet outcomes (RQ3). Answering this question furthermore validates whether communication skills add to the existing framework. Finally, this paper addresses how the support sources employed moderate the effect of skills on beneficial Internet use. Because not everyone is necessarily inclined to use support or to employ the same sources, it is possible that those who need help develop and implement Internet skills unequally. Hence, we question whether different patterns of help seeking moderate the assumed effect of Internet skills on beneficial outcomes of Internet use (RQ4).

\section{THEORETICAL BACKGROUND}

\subsection{Dimensions of Internet skills}

As explained in the introduction, the first contribution of this paper is adding communication skills to the Internet skills framework as suggested by Van Deursen \& Van Dijk $(2009,2010)$. In recent years, these skills have become increasingly important given the expanding prominence of the social Web, for instance, the influence of various social network sites (SNS), such as Facebook and Twitter. These opportunities pose difficulties of their own and require a distinct set of competences to make the most out of them. Increasingly, all sorts of existing literacy definitions are extended to account of communication skills. We follow this movement, by including a fine-grained definition to the empirically tested and validated framework.

The first instance that needs to be included in a communication skills definition relates to all sorts of social applications which require one to make and maintain a - often constantly growing - set of contacts. In a large number of social applications the Internet multiplies the number of contacts we have. To cope with this complexity and to actually engage in communication is not self-evident to many users, although it looks deceivingly simple.

Second, asynchronous message exchange services, like e-mail, Twitter or SNS, require a vast (tacit) knowledge of how encode and decode online messages. In contrast with face-toface communication, computer-mediated communication often lacks a range of natural cues that facilitate mutual understanding and guide communication dynamics. In peer-to-peer networking, it is not always clear how to address someone, when to expect a response and what to make from that response.

The third Internet communication skill is the ability to attract attention to a message. To speak on the Internet is relatively easy, whereas to actually be heard is considerably harder (Hindman, 2009). Unfortunately, many expressions on the Web are in vain. For example, a very large number of blog entries and social media messages are left unread. Successful communication involves a receiver. Thus, picking the right location to post a message and carefully considering its contents are crucial to getting your message picked up by other users, either directly or indirectly (e.g., through a search engine).

The fourth communication skill is to know how to construct a coherent online identity. This also looks deceivingly simple as SNS software offers a format that is filled in. However, 
it is not easy to create a personal online profile that stands out, reflects the self one wants to reveal and is effective in appealing to others.

The fifth communication skill is the creation of online profiles and identities, which happens during interactions with others who provide feedback. The capacity to adequately respond to feedback and to be inspired by the profiles and identities of others are important communication skills on the Internet (Jenkins, 2006). In particular, children, teens and adolescents like experimenting with virtual identities to create and to understand their unique physical and mental identities.

The final communication skill is the ability to cooperate online, which primarily rests on communication. This asks for 'the ability to identify specific functions for each member based on his or her expertise and to interact with the team members in an appropriate fashion' (Jenkins, 2006, p. 42).

The composition of operational, formal, information, communication and strategic skills are listed in Table 1. The Internet skills proposed by Van Deursen \& Van Dijk (2009, 2010) and the newly added communication skills have a conditional nature and contain gradients of difficulty. Proficiency in elementary operational and formal skills is not sufficient to benefit from the Internet in all of its aspects. The development of content-related skills requires a substrate of medium-related skills (Van Deursen, Van Dijk \& Peters, 2011). For instance, before you can evaluate the results of a search query, you need to be able to perform one, or before you can ask a question on a social network site or forum, you need to register an account through form-filling. Information and communication skills basically require the same operational and formal skills. Here, we do not consider specific communication applications (e.g., Skype) outside the Internet browser context. These applications would require additional medium-related skills. Information and communication Internet skills are needed to deploy the most complicated type of Internet skills, which are strategic skills. Taken together, the five definitions focus on technical aspects and substantive content-related issues when using the Internet.

Table 1. Conceptual definitions for Internet skills (extended from Van Deursen \& Van Dijk, 2009, 2010)

\begin{tabular}{l|l}
\hline Medium-related Internet skills \\
\hline Operational & Operating an Internet browser, meaning: \\
& Opening websites by entering the URL in a browser's location bar; \\
& Navigating forward and backward between pages using browser \\
& buttons; \\
& Saving files on a hard disk; \\
& Opening various common file formats (e.g., PDFs); \\
& Bookmarking websites; \\
& Changing a browser's preferences. \\
& Operating Internet-based search engines, meaning: \\
& Entering keywords in the proper field; \\
& Executing a search operation; \\
& Opening search results in the search result lists. \\
& Operating Internet-based form, meanings: \\
& Using the different types of fields and buttons; \\
\hline
\end{tabular}




\begin{tabular}{|c|c|}
\hline & Submitting a form. \\
\hline $\begin{array}{l}\text { Formal Internet } \\
\text { Skills }\end{array}$ & $\begin{array}{l}\text { Navigating the Internet, meaning: } \\
\text { Using hyperlinks (e.g., menu links, textual links and image links) in } \\
\text { different menu and website layouts. } \\
\text { Maintaining a sense of location when on the Internet, meaning: } \\
\text { Not becoming disoriented when navigating within a website; } \\
\text { Not becoming disoriented when navigating between websites; } \\
\text { Not becoming disoriented when opening and browsing through } \\
\text { search results. }\end{array}$ \\
\hline \multicolumn{2}{|c|}{ Content-related Internet Skills } \\
\hline $\begin{array}{l}\text { Informational } \\
\text { Internet Skills }\end{array}$ & $\begin{array}{l}\text { Locating required information by: } \\
\text { Choosing a website or search system to seek information; } \\
\text { Defining search options or queries; } \\
\text { Selecting information (on Websites or in search results); } \\
\text { Evaluating informational sources. }\end{array}$ \\
\hline $\begin{array}{l}\text { Communication } \\
\text { Internet Skills }\end{array}$ & $\begin{array}{l}\text { Communicating when on the Internet by: } \\
\text { Searching, selecting, reaching and evaluating contacts online; } \\
\text { Exchanging messages online and exchanging meaning; } \\
\text { Attracting attention online; } \\
\text { Constructing online profiles and identities; } \\
\text { Adopting alternative online identities for discovery or improvisation; } \\
\text { Pooling knowledge and exchanging meaning with others in peer-to- } \\
\text { peer networking. }\end{array}$ \\
\hline $\begin{array}{l}\text { Strategic } \\
\text { Internet Skills }\end{array}$ & $\begin{array}{l}\text { Taking advantage of the Internet by: } \\
\text { Developing an orientation toward a particular goal; } \\
\text { Taking the right actions to reach this goal; } \\
\text { Making the right decisions to reach this goal; } \\
\text { Gaining the benefits that result from this goal. }\end{array}$ \\
\hline
\end{tabular}

\subsection{Sources of support as a factor explaining Internet skill differences}

Haythornwaite (2001) stressed that communication researchers need to build a picture that situates Internet use in individuals' lives, including the people with whom they interact, the technologies that surround them and their life stages and lifestyles. In this study, we attempt to develop a more sophisticated explanation of the social and individual dynamics of the Internet in individuals' day-to-day lives by focusing on how people deal with insufficiencies in the in the former section discussed Internet skills. A first solution might be self-directed learning which entails independence in the sense that there is no need to rely on a specific source for assistance. This includes learning by doing, through trial and error, or by using self-collected instructional materials (e.g., from an online search query or through books). A second solution might be addressing others, which not only helps one discover the possibilities of the Internet, but also compensates for inadequacies in media competences (Robinson, DiMaggio \& Hargittai, 2003). Reisdorf (2011), for example, stressed the importance of proxy users; people who are not using the Internet themselves, but have someone who does things online for them. A variety of studies on information communication technologies (ICT's) have highlighted the importance of informal social networks for new media adoption (Stewart, 2007). Facer, Furlong, Furlong and Sutherland (2001), for example, 
showed that ICT skills are mainly acquired informally in homes rather than through formal education in schools, and Katz and Aspden (1997) found that most people learned how to use the Internet from family, friends and colleagues. The workplace is a frequently reported location for learning to use computers (Selwyn, Gorard \& Furlong, 2006). We consider the assistance of colleagues as a somewhat formal way of finding help, as the workplace is a more stringent professional environment that does not allow extended degrees of freedom in time or task completion. This also holds for individuals within a social network who are considered to be computer experts and whose know-how might stem from a special interest or earlier adoption of the technology than their local community (Stewart, 2007). These experts tend to be more educated, have more material resources and have a wide and heterogeneous social network (Wellman, 2001). Other sources that people with Internet skill insufficiencies might turn to are helpdesks, libraries or training. Although helpdesks usually focus on fixing a specific problem, there are also examples of (commercial) helpdesks that attempt to improve skills, mainly for seniors. Participation in guided training is a popular method for novices to develop basic skills. However, the effectiveness of training varies significantly depending on the instructional strategies employed (Cahoon, 1998). Ideally, training entails a combination of practical exercises and theory that consists of verbally presented concepts and principles. Hobbs and Frost (2003) found that media literacy education positively impacts the knowledge and understanding of media messages. Still, it remains unclear whether knowledge from media training is actually employed in everyday media consumption (Martens, 2010). In fact, it is much easier to change people's knowledge of media than to change their attitudes and actual use (Austin, Pinkleton, Hust \& Cohen, 2005; Livingstone \& Helsper, 2006). In sum, there are several ways in which people might account for Internet skill insufficiencies. However, we have no insight as to how the different types of support are combined. This leads to the first research question:

$R Q$ 1: Which combination patterns of support sources are used to address Internet skill insufficiencies?

To our knowledge, the relationship between Internet skills and the way people seek solutions for Internet skills insufficiencies has not been empirically explored in the domain of digital inequality research. Recent research with populations at large indicates that information and strategic skills leave considerable room for improvement (Van Deursen \& Van Dijk, 2011). This research also emphasized that these skills should be learned during formal education because it is unlikely that a nearby individual will be able to adequately help someone in need of instruction. Moreover, these skills do not necessarily automatically improve through increased experience or with intense use (Van Deursen \& Van Dijk, 2011; Van Deursen, Van Dijk \& Peters, 2011). In contrast, the more basic operational and formal skills are easier to learn with practice. Problems with these competences are more common among seniors and lower-educated individuals (Van Deursen \& Van Dijk, 2011; Van Deursen, Van Dijk \& Peters, 2011). Because these skills cover the more basic 'button knowledge,' it is more likely that close social contacts are able to offer simple assistance. To assess the suitability and the potential effectiveness of the support sources described, we need to gain insight into the skill 
levels of the people who tend to consult these support sources. The second research question is:

$R Q$ 2: How do the levels of Internet skills differ between people who address a specific pattern of support sources?

\subsection{Internet skills and beneficial outcomes}

As soon as the Internet became available within domestic contexts, it was clear that its use held an enormous potential for various mundane to intrusive beneficial outcomes in everyday life, ranging from getting a discount to meeting new friends. Despite early accounts of the digital divide as an issue of access, it became increasingly evident that digital skills were the decisive factor with respect to reaching such beneficial outcomes. In recent years, the digital divide has increasingly been framed as a skills divide (e.g., Hargittai, 2010; Helsper \& Eynon, 2013, Robinson et al., 2003; Van Dijk, 2005; Van Deursen \& Van Dijk, 2011; Warschauwer, 2003). Insufficient skill is a considerable barrier to being able to fully benefit from what the Internet has to offer. Unfortunately, this is rather difficult to overcome due to the problem's multidimensional, conditional structure, which was discussed in the introduction. It is not enough for people to know how to operate the Internet. To get the most out of Internet use, it is important to know how to find and evaluate information, to communicate effectively, and to understand the dynamics of what is the best means to attain a particular goal on the Internet. Possible beneficial outcomes of Internet use that are well documented in the literature cover finding jobs (e.g., Fountain, 2005), buying products cheap and obtaining discounts (e.g., Bhatnagar \& Ghose, 2004), beneficially trading goods (Bakos, 1998), finding a political party to vote for (e.g., Ward, 1996), finding and joining association or unions (e.g., Norris, 2002), making and meeting new friends (Parks \& Floyd, 1996), meeting a partner by participating in online dating (e.g., Valkenburg \& Peter, 2007), finding and identifying medical conditions (e.g., Diaz et al., 2002) and booking holidays profitable (e.g., Lang, 2000). Together, these outcomes cover all domains in which Internet use has the potential to actually matter. To obtain a better picture of the contribution of Internet skills, we propose the following research question:

\section{$R Q 3:$ Which Internet skills explain the diversity in beneficial Internet outcomes?}

We should not consider Internet users as a homogenous mass with a fixed, internally consistent configuration of skills. On a conceptual basis, we argue that skills are developed epigenetically, that is, one after the other, with increasing complexity and with strategic skills at the top. Yet, in the previous sections, we maintained the implicit hypothesis that if skills develop in such a manner, then Internet users should consult different patterns of support sources that would be ideally suited to the acquisition of specific skills. When people are not aware of what source is best for solving their problem, they might start to compensate for a particularly insufficient skill with another skill. For example, they might ask someone who knows how to operate a search engine to reduce their number of search hits, which actually requires substantial knowledge about the search query. Wrong choices can also be made by individuals who refrain from seeking help because they are comfortable using some kind of 
workaround. Although this is less likely for medium-related skills, it is very plausible for content-related skills. For example, instead of learning how to compose elaborate search queries, one could ask someone how to find something or how to assess the information available on a website. This could happen either by consulting a support source or by employing specific communication Internet skills (e.g., mobilizing a social contact to use effective messages for support questions). This also works the other way: if one fails to get responses from people online, one might compensate by employing information skills to find a source of helpful information. To summarize, two scenarios are possible: either skills are developed equally such that it does not matter which support sources are looked for, or at some point, an individual's skills start to diverge under the influence of particular support sources or as a consequence of complete self-reliance. In the former scenario, Internet skills equally explain the diversity in beneficial outcomes for all patterns of support seeking. However, if the latter scenario occurs, we encounter moderating effects of support seeking on these relations. Consequently, we added a final research question:

$R Q$ 4: Do patterns of addressing support moderate the effect of Internet skills on the diversity of beneficial outcomes?

\section{METHOD}

\subsection{Sample}

The present study draws on a sample collected in the Netherlands over a period of two weeks in September 2011 by using an online survey. To obtain a representative sample of the Dutch population, we made use of the Dutch panel of Panelclix, a professional international organization for market research, containing over 108,000 people. This panel is believed to be a largely representative sample of the Dutch population. Members receive a very small incentive of a few cents for every survey question they answer. In total, a sample of 2,850 people were randomly selected from this panel to reach a sample of about 1,200 persons. The response rate was $52 \%$, and eventually, a total of 1,482 responses were obtained. During the data collection, amendments to the sampling frame were made to be sure to represent the Dutch population in the final sample. As a result, only a very small post hoc correction was needed to correct for gender, age and education according to census data. The online survey used specific software that checked for missing responses in which users were prompted to answer them. Pretesting of the survey was conducted with ten internet users in two rounds. Amendments were made at the end of every round based on the provided feedback. No major comments were given by the ten respondents in the second round and the survey was deemed ready for posting. The time needed to answer the survey questions was reduced to about 15 minutes. Table 2 summarizes the demographic characteristics of the respondents. 
Table 2. Demographic profile $(N=1,482)$

\begin{tabular}{lc}
\hline Gender (\%) & 52 \\
Male & 48 \\
Female & 46.08 \\
\hline Age & 17.52 \\
\hline SD & \\
Education (\%) & 36 \\
Low (e.g., primary school) & 40 \\
Middle (e.g., high school) & 24 \\
High (e.g., college and university) & \\
Occupation (\%) & 42 \\
Employee & 7 \\
Employer & 4 \\
Unemployed & 6 \\
Disabled & 25 \\
Retired & 7 \\
Stay at home parent & 9 \\
Student & \\
& \\
\hline
\end{tabular}

\subsection{Measures}

The questionnaire inquired about socio-demographics, Internet skills, support sources that were sought and beneficial Internet outcomes. Internet skills were measured using an instrument proposed by Van Deursen, Van Dijk and Peters (2012). This instrument proposed a 21-item inventory for operational, formal, information and strategic Internet skills. Instead of drawing upon self-assessments, these items ask for actual behaviors that serve as indices for skills. The questionnaire's psychometric properties have repeatedly been proven to be satisfactory in terms of reliability and construct validity (i.e. convergent and discriminant validity). More specifically, the questionnaire was constructed using extensive ecologically valid skill performance field tests as benchmarks. This makes the instrument employed here more favorable when compared to the used self-assessments of skills which have significant problems of validity (e.g., Bunz, 2004; Hargittai, 2005; Merritt, Smith, \& Renzo, 2005; Talja, 2005; Van Deursen \& Van Dijk, 2010). We extended the instrument by including items that measured Internet communication skills. The six-item measure displayed a high degree of face validity. Moreover, its items loaded onto a single component and demonstrated high internal consistency. Table 3 lists the employed items.

Table 3. Descriptives and Cronbach alphas for the observed Internet skills $(\mathrm{N}=1,482)$ (five-point scale ranging from 1 (never) to 5 (daily)

\begin{tabular}{lrr}
\hline On the Internet, how often do you... & $M$ & $S D$ \\
\hline Operational Internet Skills $(\alpha=.78)$ & & \\
save files & 3.35 & 1.32 \\
use the refresh button & 2.91 & 1.64 \\
upload files to another computer & 2.10 & 1.24 \\
download programs & 2.19 & 1.13
\end{tabular}


watch video files

$3.13 \quad 1.29$

Formal Internet Skills $(\alpha=.74)$

find websites to be confusing

$2.06 \quad 1,08$

get lost

$\begin{array}{ll}1.34 & 0.68\end{array}$

feel disoriented

$\begin{array}{ll}1.57 & 0.91\end{array}$

experience difficulties with a website's layout

$2.50 \quad 1.13$

not know where you are

$1.72 \quad 1.11$

Informational Internet Skills $(\alpha=.84)$

$\begin{array}{lrl}\text { check information retrieved on another website } & 3.11 & 1.26\end{array}$

examine more than the top results

$3.83 \quad 1.19$

find the information you were looking for

$4.06 \quad 1.02$

examine the results on subsequent result pages

$1.96 \quad 0.95$

use more than one search keyword

$3.76 \quad 1.27$

Communication Internet Skills $(\alpha=.74)$

ask people for advice

$2.00 \quad 0.92$

receive positive feedback on your online profile

$2.32 \quad 1.37$

work together with others on a project

$1.79 \quad 1.24$

make new contacts

$2.19 \quad 1.05$

respond to messages in a panel discussion

$2.29 \quad 1.34$

receive feedback on posted messages

$3.17 \quad 1.37$

Strategic Internet Skills $(\alpha=.82)$

make a decision based on retrieved information

$2.98 \quad 1.20$

use information about a specific subject from multiple sites

$3.17 \quad 1.08$

benefit from using the Internet

3.391 .26

use reference Websites

$2.57 \quad 1.07$

gain financial benefits

$2.57 \quad 1.17$

Support sources were assessed using a set of eight dichotomous measures. Each item reflected a specific source that people might address when experiencing a skill insufficiency. These sources and their relative frequencies in the sample are shown in Table 4.

Beneficial outcome of Internet use were measured in a similar fashion, drawing upon ten questions with a dichotomous answering scale. The items are based on beneficial outcomes of Internet use that are well documented in the literature (see Section 2.3). Overall, the items together covered a wide range of beneficial outcomes. All items were summed into a single scale that reflects the diversity $(M=3.95, S D=2.21)$. The relative frequencies of positive responses are enumerated in Table 4.

Table 4. Descriptive statistics for support sources and Internet outcomes $(N=1,482)$

\begin{tabular}{lr} 
Support sources employed when experiencing Internet skill insufficiencies & $\%$ \\
I turn to friends or family & 33 \\
I turn to people at the library & 0 \\
I turn to people at a helpdesk & 6 \\
I turn to a computer expert & 7 \\
I turn to colleagues at work or at school & 7 \\
I turn to a formal Internet course & 21 \\
I do not need help & 59 \\
I do not know who to ask for help & 3 \\
\hline
\end{tabular}


Beneficial outcomes

I found a job after applying for an online job vacancy

I bought a product cheaper than it was in the local store

I traded or exchanged goods that I would not have sold otherwise 62

I chose which political party to vote for $\quad 34$

I found an association, club, union or party that I became a member of $\quad 22$

I got into contact with new friends who I actually met later 30

$\begin{array}{ll}\text { I actually met a potential partner who I was online dating } & 13\end{array}$

$\begin{array}{ll}\text { I found out what medical condition I was suffering from } & 27\end{array}$

$\begin{array}{ll}\text { I booked a cheaper vacation } & 61\end{array}$

I managed to obtain a discount 40

\section{RESULTS}

\subsection{Latent class analysis of support sources}

To answer the first research question, a latent class analysis (Vermunt \& Magidson, 2006) was performed on the eight independent dichotomous indicators of support sources. This technique, which is a subset of structural equation modeling, allows for the uncovering of latent structures within multivariate categorical data. The technique makes it possible to describe the aggregate responses and decompose the tabular frequencies into a set of latent classes or segments that displayed certain characteristics. A common procedure is to iterate the analysis with an increasing number of classes until a satisfactory model fit is reached. In this case, a model consisting of three classes yielded a well-fitting parsimonious solution. See Table 5.

Table 5. Summary of iterative LCA model fitting $(\mathrm{N}=1,482)$

\begin{tabular}{rrrrrrrr}
\hline $\boldsymbol{N}$ & $\boldsymbol{L} \boldsymbol{L}$ & $\mathbf{B I C}$ & $\boldsymbol{N}$ parameters & $\boldsymbol{L}^{\mathbf{2}}$ & $\mathbf{d f}$ & $\boldsymbol{p}$ & Class error \\
clusters & & & 8.00 & 1596.84 & 247 & .00 & .00 \\
1 & -3121.61 & 6299.83 & 17.00 & 340.66 & 238 & .00 & .00 \\
2 & -2493.52 & 5107.34 & 26.00 & 191.57 & 229 & .97 & .02 \\
3 & -2418.97 & 5021.94 & 35.00 & 91.71 & 220 & 1.00 & .03 \\
4 & -2369.04 & 4985.77 & & &
\end{tabular}

The retained three-class model demonstrates a very sharply delineated profile. The first cluster, labeled as 'independents', has an absolute probability of not needing any help combined with a very low probability of having had formal education. The second cluster, which consists of the socially supported, is characterized as an absolute chance of seeking support from friends and family. Finally, the third cluster of formal help seekers displays relatively high probabilities of relying on helpdesks, computer experts, colleagues and formal courses. Figure 1 summarizes indicator variables' response probabilities per class. The exact probabilities, Wald statistics and $\mathrm{R}^{2}$ indices are included in the Appendix. 


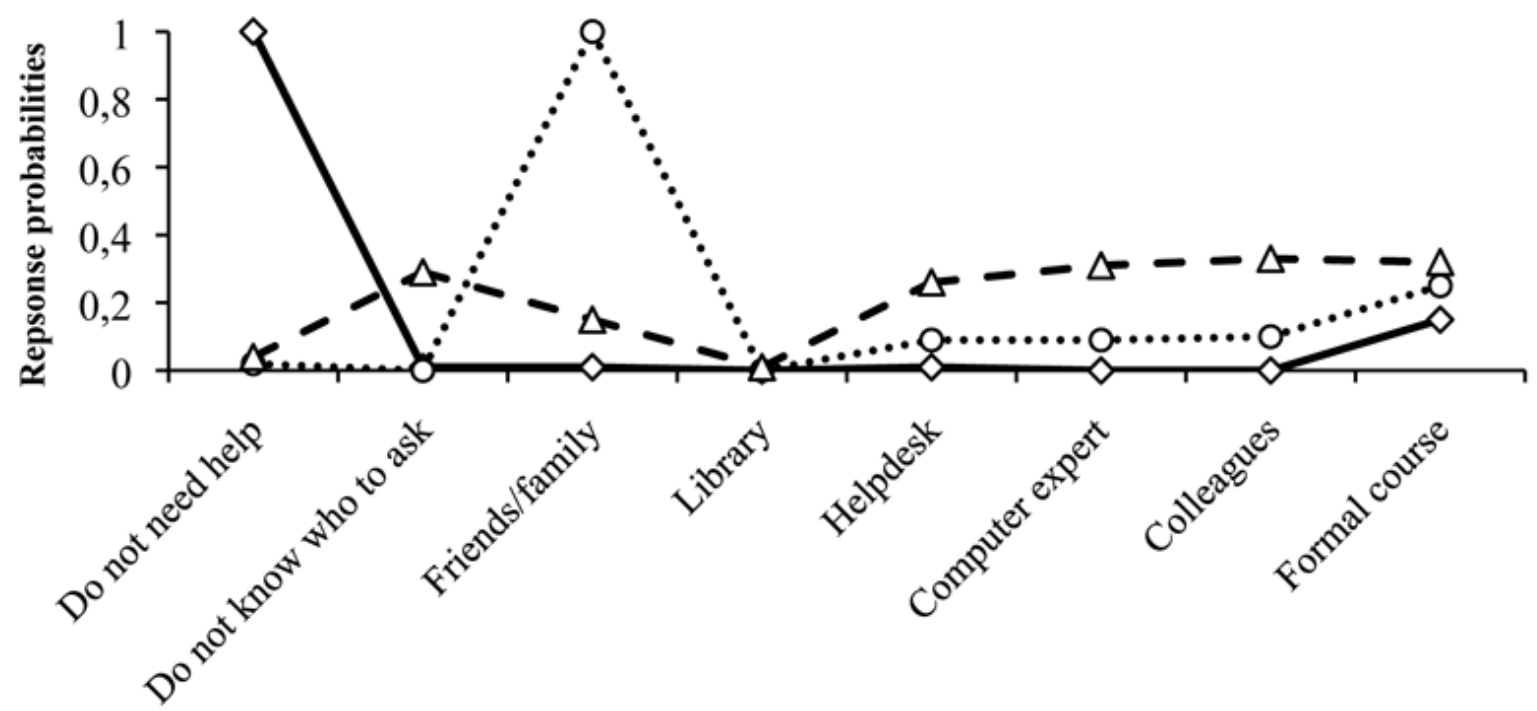

$\neg$ Cluster 1: Independents $\cdots \circ \cdot$ Cluster 2: Socially supported $-\Delta$ Cluster 3: Formal help seekers

Figure 1. Response probability plot of the retained three-class model

Table 6 shows a descriptive socio-demographic analysis which revealed that both the socially supported and formal help seekers were, on average, much older than the independents. With respect to gender, imbalances were noted for the independents, who were predominantly male, whereas the socially supported were more often female. Lower levels of education were observed for the socially supported and formal help seekers. Finally, the socially supported were more likely to be pensioners or stay-at-home parents, whereas employment rates were much higher for the formal help seekers and independents. Moreover, the latter group contained more students.

Table 6. Descriptive socio-demographic analysis of the support source clusters $(N=1,482)$

Cluster 1: $\quad$ Cluster 2: $\quad$ Cluster 3: $\quad$ Test statistic Independents Socially Formal help supported seekers

\begin{tabular}{ccccc}
\hline Age (years) & & & & $F(2,1747)=84.82 * * *$ \\
M & 42.94 & 55.44 & 52.30 & $\eta_{\mathrm{p}}{ }^{2}=.12$ \\
SD & 15.82 & 16.60 & 16.64 & \\
\hline Gender (\%) & & & & $\chi^{2}(2)=41.32 * * *$ \\
Male & 56 & 46 & 50 & \\
Female & 44 & 64 & 50 & $\chi^{2}(2)=32.27 * * *$ \\
Education level (\%) & & & & \\
Low & 30 & 49 & 37 & \\
Medium & 44 & 33 & 40 & \\
High & 26 & 18 & 23 & \\
\hline Occupational status (\%) & & & & \\
Employed & 60 & 33 & 56 & \\
Unemployed & 5 & 5 & 4 & \\
Disabled & 6 & 7 & 5 & \\
Pensioned & 12 & 36 & 25 &
\end{tabular}




\begin{tabular}{lccc} 
Stay-at-home & 5 & 12 & 7 \\
Student & 12 & 7 & 3 \\
\hline
\end{tabular}

\subsection{Multivariate analysis of Internet skills' mean structures}

The second research question concerning the mean structures of Internet skills for each support pattern was assessed using a multivariate analysis of variance. All Internet skills were simultaneously entered into a model that employs the support source clusters as a fixed factor. See Table 7. Moreover, age, gender and education (dummy coded, with the lowest level as reference) were entered as covariates. All of these covariates rendered significant effects: gender $\left(F(5,1277)=19.51, p<.001, \eta_{\mathrm{p}}{ }^{2}=.07\right)$, age $\left(F(5,1277)=89.03, p<.001, \eta_{\mathrm{p}}{ }^{2}=.26\right)$, medium education level $\left(F(5,1277)=5.65, p<.001, \eta_{\mathrm{p}}{ }^{2}=.02\right)$ and high education level $(F(5$, 1277) $\left.=24.18, p<.001, \eta_{\mathrm{p}}{ }^{2}=.09\right)$. Nonetheless, we found a significant overall effect of the support source clusters $\left(F(10,1277)=12.18, p<.001, \eta_{\mathrm{p}}{ }^{2}=.05\right)$. Furthermore, after a Bonferroni correction for Type I error, it showed that all of the mean skill levels differed except for the communication skills. Figure 2 depicts the estimated marginal means histograms. Post hoc Bonferroni contrast analysis points out that for operational skills, there is no significant difference $(p<.05)$ between the independents and formal help seekers, whereas both these groups differ from the socially supported. Both the socially supported and formal help seekers differ from the independents in terms of formal skills, albeit not differing from each other. Concerning strategic skills, the independents and formal help seekers do not differ, although they are both significantly different from the socially supported. The same is true for information skills, whereas finally, there are no significant differences for communication skills.

Table 7. Individual tests of between-subjects effects, employing support source clusters as a fixed factor and Internet skills as dependent variables $(\mathrm{N}=1,482)$

\begin{tabular}{lccc}
\hline Dependent Variables & $F(2,1281)$ & $p$ & $\eta_{\mathrm{p}}{ }^{2}$ \\
\hline Operational skills & 9.63 & .000 & .02 \\
Formal skills & 11.88 & .000 & .03 \\
Informational skills & 17.68 & .000 & .03 \\
Communication skills & .49 & .612 & .00 \\
Strategic skills & 5.54 & .004 & .01 \\
\hline
\end{tabular}

The marginal means histograms in Figure 2 demonstrate that the independents consistently had the highest skill levels. However, there were no significant differences in formal skills among the socially supported and in strategic skills when compared to the formal help seekers. Members of the latter cluster displayed the second highest levels of operational, strategic and information skills. However, the socially supported had stronger formal skills. 


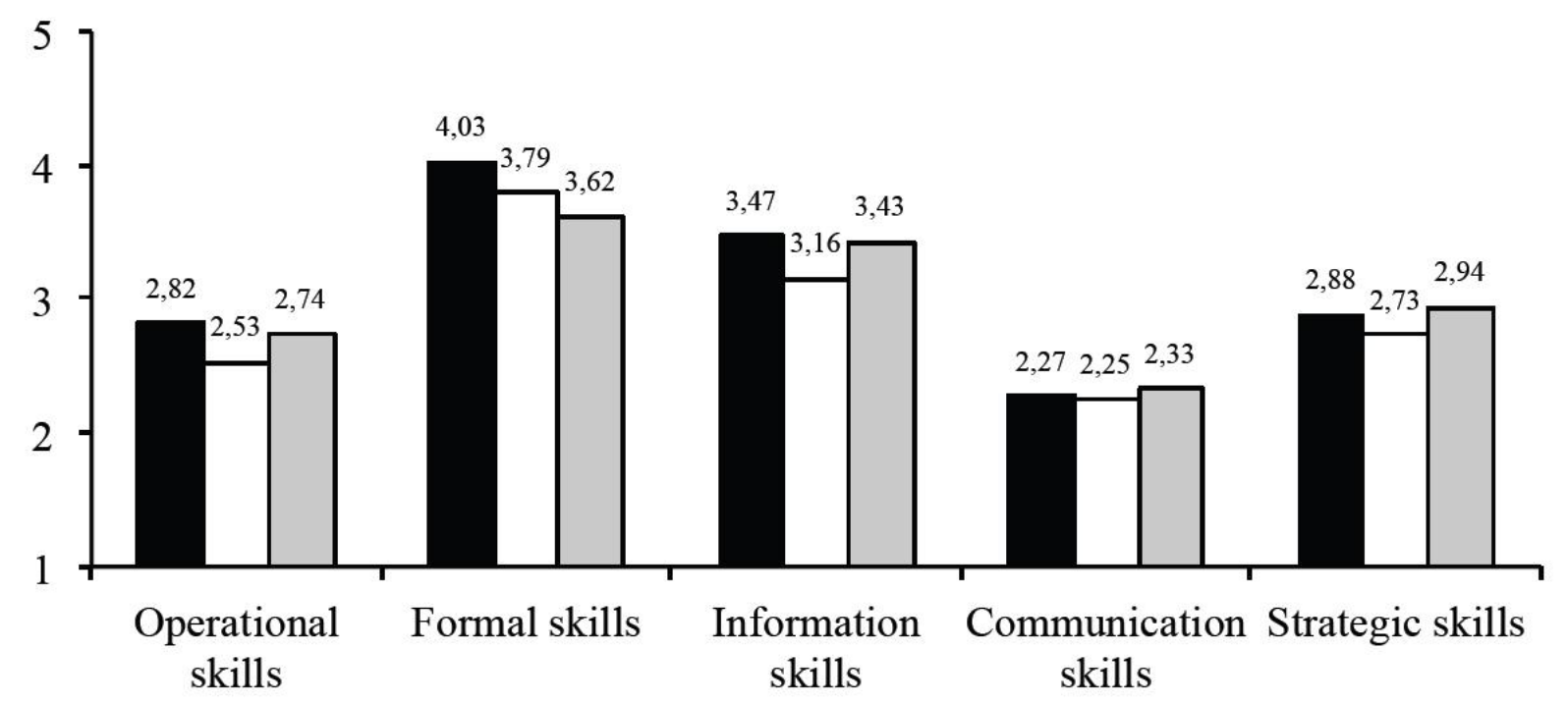

שCluster 1: Independents $\mathbf{a}$ Cluster 2: Socially supported aCluster 3: Formal help seekers

Figure 2. Marginal means histograms of three support sources clusters' scores on Internet skills according to a multivariate analysis of variance

\subsection{Structural relations between Internet skills and beneficial Internet use}

In light of the third and fourth research questions, we investigated the structural relationships between Internet skills on one hand, and beneficial outcomes of Internet use on the other hand. With regard to Internet skills, the cluster of independents significantly differed from the socially supported and the formal help seekers in terms of the level of attained beneficial outcomes $\left(F(2,1479)=16.34, p<.001, \eta_{\mathrm{p}}{ }^{2}=.02\right)$; The independents scored a marginal mean of 4.18 , the socially supported 3.44 , and the formal help seekers 3.59 out of a total of 10 effects.

We are equally interested in the correlational structures between the aforementioned variables. To investigate this matter, a stepwise regression model was computed that employed the beneficial Internet outcomes as dependent variables and Internet skills as independent variables. Because the final model contained interaction terms, the independent variables were centered first, which involves subtracting the mean from all of the variables' data points to avoid excessive multicolinearity when computing interaction terms (Aiken, West \& Reno, 1991). In the first block, the centered existing skill variables were entered (i.e. operational, formal, information and strategic skills). The second block added the centered measure of communication skills. In the third block, interaction effects with the third cluster were added. In the fourth and final block, interaction effects with the third cluster were entered. As such, we were able to assess the moderation effects of cluster membership on the relation between skills and outcomes of Internet use. The final model showed a good fit $(F(15,1466)=181.79, p<.001)$, explaining $39 \%$ of the variance in beneficial outcomes. See Table 8. 
Table 8. Regression model of positive Internet outcome effects of Internet skills $(\mathrm{N}=1,482)$. The summarized estimates are those of the final step.

\begin{tabular}{clrrrr}
\hline Block & Independent variables & $\beta$ & $t$ & $p$ & $\mathrm{R}^{2}$ \\
\hline 1 & Operational skills & .12 & 2.92 & .004 & .36 \\
& Formal skills & -.02 & -.75 & .456 & \\
& Informational skills & -.05 & -1.18 & .240 & \\
& Strategic skills & .38 & 9.54 & .000 & \\
\hline 2 & Communication skills & .26 & 7.79 & .000 & .38 \\
\hline 3 & Cluster 2 x Operational skills & .05 & 1.41 & .158 & .39 \\
& Cluster 2 x Formal skills & .03 & 1.02 & .308 & \\
& Cluster 2 x Informational skills & .07 & 1.62 & .105 & \\
& Cluster 2 x Strategic skills & -.04 & -.97 & .332 & \\
& Cluster 2 x Communication skills & -.08 & -2.53 & .011 & \\
\hline \multirow{2}{*}{4} & Cluster 3 x Operational skills & .02 & .55 & .580 & .39 \\
& Cluster 3 x Formal skills & .03 & .97 & .334 & \\
& Cluster 3 x Informational skills & .01 & .32 & .753 & \\
& Cluster 3 x Strategic skills & -.01 & -.13 & .894 & \\
& Cluster 3 x Communication skills & -.03 & -1.10 & .272 & \\
\hline
\end{tabular}

Note: All estimates demonstrate satisfactory collinearity diagnostics: tolerance > .20, variance-inflation factor $<5$ [24].

The final model shows significant effects of operational $(\beta=.12, p<.05)$, strategic $(\beta=.38$, $p<.001)$ and communication skills $(\beta=.26, p<.001)$ on beneficial outcomes. Interestingly, communication skills independently explain variance in beneficial outcomes, unaccounted for by the other skills. This pattern was remarkably robust for all of the three clusters. We encountered only one significant interaction effect for communication skills in the second block $(\beta=-.08, p<.05)$. This estimate, however marginal in size, indicates that, in comparison with the cluster of independents, the socially supported shared a smaller effect of communication skills on beneficial outcomes, rendering it a slightly less important explanatory factor.

\section{DISCUSSION}

\subsection{Main findings}

Patterns of support seeking are important reactions to the complexities many people experience when using the Internet. In light of research question 1, we conclude that three emerging support patterns appear and reveal a remarkably sharp delineation. First, a large majority behaves as if they were completely self-reliant. This group contains more males than females and more medium and highly educated people than lower educated people. A second pattern consists of Internet users who rely on their direct, informal social network of family and friends. This group contains more females and lower educated people. A third pattern reveals a group of formal help seekers who combine sources such as help desks, colleagues, computer experts and courses. These are addressed more by lower and medium educated Internet users than highly educated users. 
Research question 2 addressed the relation between the three patterns of support sources and the five types of Internet skills. It appears that the self-reliant Internet users scored the highest on all Internet skills: operational, formal, information, communication and strategic skills. The second highest scorers were the Internet users who rely on more formal support sources and the lowest scores were for the seekers of social support of friends and family. These scores indicate that the most natural and informal solution for people - to mobilize their social network - does not sufficiently help them to catch-up with those who already claim to have a high level of skills and those who seek formal help from courses, books, help desks and experts. From the literature overview, we argued that the social support of friends and family would be suitable to compensate for a lack of medium-related skills, whereas more formal support sources assist in difficulties with content-related skills. Those individuals who sought help from more formal sources had indeed stronger information and strategic skills. However, those individuals who addressed insufficient Internet skills by turning to friends and family had lower levels of operational skills. Thus, seeking the support of family and friends is not related to stronger operational skills. This raises questions about the quality and effectiveness of this source of help, although not using these sources might be even less effective. Learning operational skills remains a high priority for Internet users, particularly for beginners. Self-reliant Internet users consistently demonstrate the highest skill scores, which suggests that these people indeed share a low need for support, provided that our skill measures observed the frequency of this behavior, rather than self-evaluation.

In this investigation we added communication skills to the skill definition of Van Deursen \& Van Dijk (2010). We conceptualized Internet skills as being acquired through a gradual process, starting with operational and formal skills that evolve into more established information and communication skills and are complete with the attainment of strategic skills. The third research question addressed which of these skills actually matter for attaining beneficial outcomes from the Internet. It appears that the newly added communication skills are an important set of skills. Mastering these skills provides users with more chances on the Internet. We have seen that people generally rely more on Internet communication skills than on Internet information skills to attain beneficial outcomes and to steadily develop more advanced strategic skills. It is very likely that this is due to the direction of development that the Internet has taken in recent years. Social media, such as social networking sites, have been persistently growing. Our research clearly indicates that the evolution towards a social web, in which communication skills are becoming increasingly important, will be accompanied by greater diversity in beneficial outcomes. Furthermore, Internet communication skills hold the potential for achieving a high degree of independence when using the Internet for one's own goals. These communication skills are able to compensate for a lack of information skills. Notably, operational skills still yield a positive explanation for beneficial outcomes, even when content-related skills were entered into the equation. This implies that there is still conspicuous variation in the level of operational skills, at least in the Dutch population. Presumably, operational skills are a primary condition for using and benefiting from the Internet at a basic level. Altogether, our study shows that Internet skills matter since three of 
the five Internet skills have a significant effect on the beneficial outcomes when using the Internet.

The consequence of the unequal distribution of skills and of the different use of support sources is an unequal distribution of the benefits the Internet has to offer. Concerning Research Question 4, we can conclude that self-reliant Internet users benefit more from their Internet communication skills than those individuals who rely on friends and family (there was no difference between self-reliant Internet users and those seeking formal help). This implies that self-reliant Internet users seem to utilize the Internet's social features to benefit from this medium. Moreover, it is possible that they are actually self-reliant given their competence in communicating effectively through the Internet, either by addressing its broad communities to obtain directions or by learning more about beneficial outcomes. In contrast, those individuals who seek social support to compensate for a lack of skills might seek the same information by simply asking the people who immediately surround them. It is an important finding that Internet communication skills hold the potential for providing a path to advanced strategic skills, and in doing so, they actually afford a certain degree of independence when using the Internet. Furthermore, in comparison with information skills, communication skills are less cognitively demanding. It seems worthwhile to invest more resources into the development of Internet communication skills, for example, through formal education.

\subsection{Shortcomings and future research}

The relationships between Internet skills and sources of support sought as well as between Internet skills and being able to take advantage of the opportunities the Internet has to offer have, to our knowledge, never been explicitly investigated. In this study we attempted to shed more light on these relationships. Patterns of support seeking are important reactions to the complexities many people experience when using the Internet. Although the nature of this research was exploratory and can only reveal results for one country, it does show important patterns of support seeking and their effects on taking advantage of the Internet. Considering the general nature of the conceptual apparatus used in this study, there is no reason to think that the results of this study would only apply to the Netherlands.

Using surveys to measure Internet skills has several problems of validity. However, for measuring operational, formal, information and strategic Internet skills we used measures that have repeatedly been proven to be satisfactory in terms of reliability and validity. More specifically, the items for measuring Internet skills were tested with extensive, ecologically valid skill performance field tests as benchmarks. In this contribution, we extended this instrument by including items that measured Internet communication skills. Although these skills have not been validated by using field tests, the six-item measure displayed a high degree of face validity. Moreover, its items loaded onto a single component and demonstrated high internal consistency. Future research however, should further validate these items by conducting field tests. They might also be extended by including other aspects related to online communication, not accounted for in this contribution. 
Furthermore, this study shows the need for future research concerning how Internet communication skills are actually employed and, in particular, how they relate to Internet information skills.

Regarding the beneficial outcomes it is recommended to further investigate such outcomes so that a wider range can be included in future studies. Here, we added beneficial outcomes that are acknowledged in a wide range of studies. In future studies, however, a more theoretically investigation of several participation areas should be made so that the outcomes can evenly be distributed over these areas.

\section{REFERENCES}

Aiken, L.S., West, S.G. \& Reno, R.R. (1991). Multiple Regression: Testing and interpreting interactions. London: Sage.

Austin, E.W., Pinkleton, B.E., Hust, S.J.T. \& Cohen, M. (2005). Evaluation of an American Legacy Foundation. Health Communication, 18(1), 75-95.

Ba, H., Tally, W. \& Tsikalas, K. (2002). Investigating children's emerging digital literacies. Journal of Technology, Learning and Assessment, 1(4), 1-48.

Bakos, C. (1998). The emerging role of electronic marketplaces on the Internet. Communications of the ACM, 41(8), 35-42.

Bhatnagar, A. \& Ghose, S. (2004). Segmenting consumers based on the benefits and risks of Internet shopping. Journal of Business Research, 57(12), 1352-1360.

Bunz, U. (2004). The computer-Email-Web (CEW) Fluency Scale-Development and validation. International Journal of Human-Computer Interaction, 17, 479-506.

Cahoon, A. (1998). Teaching and learning Internet skills. New directions for Adult and Continuing Education, 78, 5-13.

Diaz, J.A., Griffith, R.A., Ng, J.J., Reinert, S.E., Friedmann, P.D. \& Moulton, A.W. (2002). Patients' Use of the Internet for Medical Information. Journal of General Internal Medicine, 17(3), 180-185.

Facer, L., Furlong, J., Furlong, R. \& Sutherland, R. (2001). What's the point of using computers? The development of young people's computer expertise in the home. New Media and Society, 3(2), 199-219.

Fountain, C. (2005). Finding a Job in the Internet Age. Social Forces, 83(3), 1235-1262.

Hargittai, E. (2005). Survey measures of web-oriented digital literacy. Social Science Computer Review, 23(3), 371-379.

Hargittai, E. \& Hinnant, A. (2008). Digital Inequality, differences in Young Adults' Use of the Internet. Communication Research, 35(5), 602-621.

Hargittai, E. (2010). Digital Na(t)ives? Variation in Internet Skills and Uses among Members of the Net Generation. Sociological Inquiry, 80(1), 92-113.

Helsper, E.J. \& Eynon, R. (2013). Distinct skill pathways to digital engagement. European Journal of Communication. doi: 10.1177/0267323113499113

Haythornthwaite, C. (2001). Introduction: the internet in everyday life. American Behavioral Scientist, 43(3), 363-382. 
Hindman, M. (2009). The Myth of Digital Democracy. Princeton, NJ: Princeton University Press.

Hobbs, R. \& Frost, R. (2003). Measuring the acquisition of media literacy skills. Reading Research Quarterly, 38(3), 330-355.

Jenkins, H. (2006). Confronting the challenges of participatory culture: Media education for the 21st century. The MacArthur Foundation.

Katz, J. \& Aspden, P. (1997). Motivations for and barriers to internet usage: results of a national public opinion survey. Internet Research, 7(3), 170-188.

Lang, T.C. (2000). The effect of the Internet on travel consumer purchasing behaviour and implications for travel agencies. Journal of Vacation Marketing, 6(4), 368-385.

Lankshear, C. \& Knobel, M. (2008). Digital literacies: Concepts, policies and practices. New York: Peter Lang.

Linvingstone, S. (2008). Internet literacy: Young people's negotiation of new online opportunities. Digital youth, innovation, and the unexpected. In T. McPherson (ed). Digital media and learning (pp. 101-122). Cambridge, MA: MacArthur Foundation.

Livingstone, S. \& Helsper, E. (2006). Does advertising literacy mediate the effects of advertising on children? A critical examination of two linked research literatures in relation to obesity and food choice. Journal of Communication, 56(3), 560-584.

Martens, H. (2010). Evaluating media literacy education: concepts, theories and future directions. Journal of Media Literacy Education, 2(1), 1-22.

Merritt, K., Smith, D. \& Renzo, J.C.D. (2005). An investigation of self-reported computer literacy: Is it reliable? Issues in Information Systems, 6(1), 289-295.

Mossberger, K., Tolbert, C.J. \& Stansbury, M. (2003). Virtual Inequality: Beyond the Digital Divide. Georgetown University Press.

Norris, P. (2001) Digital Divide: Civic Engagement, Information Poverty and the Internet Worldwide. Washington, DC: Cambridge University Press.

Norris, P. (2002). The Bridging and Bonding Role of Online Communities. Press/Politics, 7(3), 3-13.

Parks, M.R. \& Floyd, K. (1996). Making Friends in Cyberspace. Journal of ComputerMediated Communication, 1, pp. 0.

Reisdorf, B.C. (2011). Non-adoption of the internet in great britain and Sweden. A crossnational comparison. Information, Communication \& Society, 14(3), 400-420.

Robinson, J., DiMaggio, P. \& Hargittai, E. (2003). New social survey perspectives on the digital divide. IT \& Society, 1(5), 1-22.

Selwyn, N., Gorard, S. \& Furlong, J. (2006). Adult learning in the digital age: information technology and the learning society. New York: Routledge.

Solomon, G., Allen, N.J. \& Resta, P. (2003) Toward digital equity: Bridging the divide in education. Boston, MA: Pearson Education Group.

Stewart, J. (2007). Local experts in the domestication of information and communication technologies. Information, Communication \& Society, 10(4), 547-569.

Talja, S. (2005). The social and discursive construction of computing skills. Journal of the American Society for Information Science and Technology, 56(1), 13-22. 
Valkenburg, P.M. \& Peter, J. (2007). Who Visits Online Dating Sites? Exploring Some Characteristics of Online Daters. CyberPsychology \& Behavior, 10(6), 849-852.

Van Deursen, A.J.A.M. \& Van Dijk, J.A.G.M. (2009). Using the Internet: Skill Related Problems in Users' Online Behavior. Interacting with Computers, 21, 393-402.

Van Deursen A.J.A.M. and Van Dijk J.A.G.M. (2010). Measuring internet skills. International Journal of Human-Computer Interaction, 26(10), 891-916.

Van Deursen, A.J.A.M. \& Van Dijk, J.A.G.M. (2011). Internet Skills and the Digital Divide. New Media \& Society, 13(6), 893-911.

Van Deursen, A.J.A.M., Van Dijk, J.A.G.M. \& Peters, O. (2011). Rethinking Internet skills. The Contribution Of Gender, Age, Education, Internet Experience, And Hours Online To Medium- And Content-Related Internet Skills. Poetics, 39, 125-144.

Van Deursen, A.J.A.M. Van Dijk, J.A.G.M. \& Peters, O. (2012). Proposing a survey instrument for measuring operational, formal, information and strategic Internet skills. International Journal of Human-Computer Interaction, 28(12), 827-837.

Van Dijk, J. (2005). The deepening divide. Inequality in the information society. London: Sage Publications.

Vermunt, J.K. \& Magidson, J. (2006). Latent Class Analysis, retrieved from: http://www.statisticalinnovations.com/articles/Latclass.pdf

Ward, M. (1996). The Effect of the Internet on Political Institutions. Industrial and Corporate Change, 5(4), 1127-1141.

Warschauer, M. (2003). Technology and Social inclusion: Rethinking the Digital Divide. Cambridge, MA: The MIT Press.

Wellman, B. (2001). Physical place and cyberplace: the rise of personalized networking. International Journal of Urban and Regional Research, 25(2), 227-252. 


\section{APPENDIX}

\begin{tabular}{lrrrrrr}
\hline & $\begin{array}{c}\text { Cluster 1: } \\
\text { Independents }\end{array}$ & $\begin{array}{c}\text { Cluster 2: } \\
\text { Socially } \\
\text { supported }\end{array}$ & $\begin{array}{c}\text { Cluster 3: } \\
\text { Formal help } \\
\text { seekers }\end{array}$ & Wald & $\boldsymbol{p}$ & $\mathbf{R}^{\mathbf{2}}$ \\
\hline Do not need help & 1.00 & .02 & .04 & 18.73 & .000 & .96 \\
Do not know who to ask & .01 & .00 & .29 & 53.57 & .000 & .23 \\
Friends/family & .01 & 1.00 & .15 & 23.68 & .000 & .92 \\
Library & .00 & .00 & .01 & 0.77 & .680 & .01 \\
Help desk & .01 & .09 & .26 & 49.25 & .000 & .11 \\
Computer expert & .00 & .09 & .31 & 54.52 & .000 & .15 \\
Colleagues & .00 & .10 & .33 & 58.25 & .000 & .16 \\
Formal course & .15 & .25 & .32 & 26.77 & .000 & .02 \\
\hline
\end{tabular}

LCA's exact response probabilities, Wald statistics and $\mathbf{R}^{2}$. The Wald statistics' magnitudes reflect the relative importance of an indicator in distinguishing between clusters, the higher the Wald and its respective $R^{2}$ value, the more important. 


\section{Biographies:}

Alexander J.A.M. van Deursen is an Assistant Professor at the Department of Media, Communication and Organization of the University of Twente in the Netherlands. His research focuses on digital inequality with specific attention to Internet skills.

Cédric Courtois (MSc, $\mathrm{PhD})$ is a senior researcher at the iMinds research group for Media and ICT, at Ghent University, Belgium. His research interests concern the social circumstances and consequences of digital media consumption, online prosuming, and youth and new media.

Jan A.G.M. van Dijk is Professor of Communication Science and the Sociology of the Information Society at the University of Twente, the Netherlands. He is chair of the Department of Media, Communication and Organization and Director of the Center for eGovernment Studies at the same university. 